\title{
Variaciones de la frecuencia cardíaca, temperatura corporal y presión arterial en pacientes sometidos a cirugía de implantes dentales
}

\author{
Variations in heart rate, body temperature and blood pressure \\ in patients undergoing dental implant surgery \\ Variações na freqüência cardíaca, temperatura corporal e pressão \\ arterial em pacientes submetidos à cirurgia de implante dentário \\ Antony Quinto Argote ${ }^{1}$ ORCID: 0000-0001-6048-5845 \\ Lourdes Rodríguez Olivos² ORCID: 0000-0001-9875-991X \\ Sixto Grados Pomarino ${ }^{3}$ ORCID: 0000-0002-4404-5230 \\ Yuri Castro-Rodríguez ORCID: 0000-0002-9587-520X
}

\section{Resumen}

Objetivo: Determinar las variaciones de la frecuencia cardiaca, presión arterial y temperatura en pacientes con cirugía de implantes dentales. Métodos: Serie de casos clínicos que incluyó a 26 pacientes de 20 a 60 años de edad atendidos en el Centro Médico Naval "Cirujano Mayor Santiago Távara" (Lima. Perú). Se registró la temperatura, frecuencia cardiaca y presión arterial 5 min antes de la cirugía de implantes, 5 min después de la anestesia local y 5 minutos después de la cirugía. Para realizar las mediciones se utilizó un termómetro digital y un monitor digital de muñeca. Resultados: La temperatura corporal durante el preoperatorio fue de 36,9 $\pm 0,3$ ${ }^{\circ} \mathrm{C}$ mientras que en el posoperatorio fue de $36,7^{\circ} \mathrm{C} \pm 0,07(p>0,05)$. La frecuencia cardiaca aumentó durante el intraoperatorio $(80,3 \pm 8 \mathrm{lat} / \mathrm{min})$ y se restableció en el posoperatorio $(72,5 \pm 7,4 \mathrm{lat} / \mathrm{min})(p<0,05)$. La presión arterial media en el intraoperatorio en promedio fue $100,14 \pm 6,35 \mathrm{mmHg}$ mientras que en el posoperatorio fue 93,97 \pm 7,57 $\mathrm{mmHg}(p<0,05)$. Conclusión: Las principales variaciones se encontraron desde el momento intraoperatorio hasta el posoperatorio en cuanto a la frecuencia cardiaca, presión arterial media, sistólica y diastólica; siendo parámetros relevantes para la consideración clínica.

Palabras clave: presión arterial, frecuencia cardíaca, temperatura corporal, implantes dentales.

\footnotetext{
Cirujano Dentista. Sociedad Científica de Estudiantes de Odontología. Universidad Nacional Mayor de San Marcos. Lima, Perú. Cirujano Dentista. Facultad de Odontología. Universidad Nacional Mayor de San Marcos. Lima, Perú.

Cirujano Dentista. Especialista en Periodoncia. Facultad de Odontología. Universidad Nacional Mayor de San Marcos. Lima, Perú.

Cirujano Dentista. Magíster en Educación. Facultad de Odontología. Universidad Nacional Mayor de San Marcos. Lima, Perú.
}

Fecha de recibido: 22/7/2020 - Fecha de aceptado: 29/9/2020 


\section{Abstract}

Objective: Determine the variations of heart rate, blood pressure and temperature in patients with dental implant surgery. Methods: Series of clinical cases that included 26 patients from 20 to 60 years of age assisted at the Naval Medical Center "Cirujano Mayor Santiago Távara" (Lima, Peru). The temperature, heart rate and blood pressure were recorded 5 minutes before implant surgery, 5 minutes after local anesthesia and 5 minutes after surgery. To make the measurements, a digital thermometer and a digital wrist monitor were used. Results: The body temperature during the preoperative period was $36.9 \pm$ $0.3^{\circ} \mathrm{C}$ while in the intra and postoperative period it was $36.7 \pm 0.07^{\circ} \mathrm{C}(\mathrm{p}>0.05)$. The heart rate increased during the intraoperative period $(80.3 \pm 8$ beats $/ \mathrm{min})$ and was restored in the postoperative period (72.5 \pm 7.4 beats $/ \mathrm{min})(\mathrm{p}<0.05)$. The mean arterial pressure in the intraoperative on average was $100.14 \pm 6.35 \mathrm{mmHg}$ while in the postoperative period it was $93.97 \pm 7.57$ $\mathrm{mmHg}(\mathrm{p}<0.05)$. Conclusion: The main variations were found from the intraoperative to the postoperative moment in terms of heart rate, mean, systolic and diastolic blood pressure; being relevant parameters for clinical consideration.

Keywords: arterial pressure, heart rate, body temperature, dental implants.

\section{Introducción}

La cirugía de implantes puede ser complementada con tratamientos adicionales como exodoncias, elevación de piso de seno maxilar, injertos de tejidos blandos, etc. Mientras se realizan estos procedimientos se presentan sucesos como estrés, ansiedad, miedo, entre otros. De-

\section{Resumo}

Objetivo: Determine as variaçóes na freqüência cardíaca, pressão arterial e temperatura em pacientes com cirurgia de implante dentário. Métodos: Uma série de casos clínicos que incluíram 26 pacientes de 20 a 60 anos de idade atendidos no Centro Médico Naval "Prefeito Cirurgiāo Santiago Távara" (Lima, Peru). Temperatura, freqüência cardíaca e pressão arterial foram registradas 5 minutos antes da cirurgia do implante, $5 \mathrm{mi}$ nutos após a anestesia local e 5 minutos após a cirurgia. Para a realização das medidas, foram utilizados termômetro digital e monitor de pulso digital. Resultados: A temperatura corporal no período pré-operatório foi de $36,9 \pm 0,3^{\circ} \mathrm{C}$, enquanto no pós-operatório foi de $36,7^{\circ} \mathrm{C} \pm 0,07$ ( $\left.p>0,05\right)$. A frequência cardíaca aumentou no intraoperatório $(80,3$ \pm 8 batimentos / $\mathrm{min}$ ) e foi restaurada no pós -operatório $(72,5 \pm 7,4$ batimentos / $\mathrm{min}$ ) (p $<0,05)$. A pressão arterial média no período intraoperatório foi em média de 100,14 \pm $6,35 \mathrm{mmHg}$, enquanto no pós-operatório foi de 93,97 $\pm 7,57 \mathrm{mmHg}(\mathrm{p}<0,05)$. Conclusão: As principais variações foram encontradas no momento intra-operatório para o pós-operatório em termos de frequência cardíaca, média, pressão arterial sistólica e diastólica; sendo parâmetros relevantes para consideração clínica.

Palavras-chave: pressão arterial, freqüência cardíaca, temperatura corporal, implantes dentários.

bido a esto, los procedimientos podrían asociarse a una intensa respuesta cardiovascular, con frecuencia inocua en pacientes saludables, pero potencialmente dañina en individuos con dolencias cardíacas.

La elevación de los procesos simpáticos que se asocia al estrés, podría eventualmente, desencadenar una respuesta cardíaca desfavorable, de- 
bido a que se elabora una elisión vagal y una excitación alfa beta adrenérgica pudiendo provocar una isquemia miocárdica por elevación del gasto cardiaco, y también posiblemente causar un descenso de la función ventricular izquierda ${ }^{(1)}$. Aquí son importantes el conocimiento de las respuestas fisiológicas sistémicas que las intervenciones quirúrgicas orales como la colocación de implantes dentales puedan provocar. Los niveles de catecolaminas circulantes se pueden elevar en eventos de estrés quirúrgicos manifestando un incremento de la presión sanguínea (PA) a parámetros perjudiciales para la persona ${ }^{(2,3)}$.

La manifestación que se produce en el organismo ante los estímulos estresores son conocidos como respuestas fisiológicas. Debido a ello, el organismo tiene una serie de reacciones fisiológicas activando el sistema nervioso vegetativo y el eje hipofisosuprarrenal ${ }^{(4)}$. Ambos sistemas generan sustancias elaboradas en las glándulas que, transportadas a través de la sangre, inhiben, excitan o regulan la actividad de los órganos (hormonas) ${ }^{(4)}$. Estas respuestas fisiológicas pueden afectar mucho más aún si ocurren en pacientes medicamente comprometidos ya que estos pacientes presentan mayor riesgo de interacciones entre la enfermedad y la cirugía de implante implicando un mayor riesgo médico (5).

A través de un correcto diagnóstico y planificación de cada paciente a tratar, los implantes dentales son tratamientos que pueden ocasionar dolor o molestias que son controlables y pasajeras. Es importante evaluar la presión arterial, temperatura y frecuencia cardíaca en cirugías a nivel odontológico. Se conoce que un aumento de la frecuencia cardiaca se asocia con un mayor riesgo de enfermedad cardiaca. Del mismo modo temperaturas desmesuradamente elevadas o disminuidas pueden causar deterioro en los tejidos, perturbar la función de órganos o alterar los índices metabólicos. Por otra parte, una crisis hipertensiva debido al aumento de la presión arterial puede causar un accidente cerebro vascular a nivel encefálico o un infarto agudo al miocardio.

El acto quirúrgico al realizar los implantes dentales modifica las respuestas fisiológicas en los pacientes ya que al estar sometidos frente a estímulos estresores en este caso el acto quirúrgico se liberan hormonas. Estas respuestas fisiológicas mantenidas provocarán un conjunto de síntomas como: hipertensión arterial, dolor precordial, aumento de la temperatura corporal, desórdenes estomacales (indigestión, diarrea), insomnio y ansiedad, sequedad en la boca y garganta, tensión y dolor muscular ${ }^{(4)}$. Aunque no es frecuente la aparición de tales síntomas durante la colocación de implantes dentales es importante considerar si las respuestas fisiológicas sistémicas se ven afectadas durante los momentos quirúrgicos de la colocación del implante; esto permite conocer si tales parámetros se ven afectados por lo que requerirán un mejor control del paciente y la situación quirúrgica. De esta forma en la presente investigación se valoró si las respuestas fisiológicas (temperatura corporal, presión arterial y frecuencia cardíaca) se ven alteradas antes, durante y después de la colocación de implantes dentales.

\section{Método}

Se diseñó un estudio observacional y descriptivo (serie de casos clínicos) con múltiples mediciones a un mismo objeto de estudio (tipo longitudinal). La población incluyó a pacientes adultos de atendidos en el Servicio de Periodoncia del Centro Médico Naval "Cirujano Mayor Santiago Távara" (Lima. Perú) desde junio a diciembre del 2018. El tamaño muestral fue determinado a partir de la estimación de medias teniendo en consideración un nivel de confianza del 95\%, un error de precisión del 5\% y una varianza esperada de 13,02 obtenido del estudio de Carrasco y cols ${ }^{(6)}$ para la estimación de la variación en la presión arterial. Según el cálculo se requirió 25 pacientes como mínimo. 
La selección muestral se realizó a través de un muestreo sistemático teniendo una constante de evaluación de $\mathrm{K}=2$. El listado de pacientes planificados para la colocación de implantes dentales fue suministrado por la Sala de Operaciones del Departamento de Estomatología del hospital. Los pacientes debieron cumplir los criterios de ser adultos entre 20 a 60 años de edad, estar planificados para la colocación máxima de dos implantes dentales y presentarse en buena condición sistémica (ASA I). Se excluyeron pacientes con afeccione sistémicas, antecedentes de intervenciones quirúrgicas, estar bajo prescripción farmacológica, ser edéntulos totales y haber tenido la experiencia de cirugías bucomaxilofaciales.

Todos los pacientes fueron atendidos por el mismo especialista en Periodoncia e Implantología Oral, se utilizó como anestésico local a la lidocaína 1: 80000 (Scandicaine ${ }^{\oplus}$, Austria) con epinefrina (máximo tres cartuchos; pasado esa cantidad el paciente fue excluido del estudio). Cinco minutos antes de iniciar la entrada a sala de operaciones a los pacientes se les pidió tomar asiento y levantar la lengua para introducir el termómetro digital y cerrar la boca de forma normal, sin apretar los dientes o los labios y se esperó un minuto para retirarlo (emitió un sonido cuando haya finalizado la toma). Se tomó el registro de la temperatura con un termómetro digital (Citizen ${ }^{\oplus}$, Japón) asegurándonos que no haya ninguna medición previa en la pantalla. Luego del registro de temperatura se procedió a evaluar la frecuencia cardiaca y la presión arterial con un monitor digital de muñeca marca (Citizen ${ }^{\circledR}$, Japón) (Fig. 1A). El paciente estuvo sentado y colocó su codo en una mesa, se envolvió el puño de presión sobre la muñeca verificando que el monitor esté en una correcta posición.

Cuando se colocó los campos estériles simples y fenestrados, se realizó la asepsia y antisepsia y la colocación de anestesias de lidocaína con vasoconstrictor 1: 80000 (Scandicaine ${ }^{\circledR}$, Austria) se esperó 5 minutos para volver hacer el registro con el termómetro digital y el monitor digital de muñeca; por comodidad del paciente y del operador se registró acostado al paciente en la cama de operaciones (Fig. 1B). Terminada la cirugía de los implantes dentales se esperó 5 minutos mientras el paciente se reincorporaba y se sentaba en la sala de cirugía para realizar un último registro con el termómetro digital y monitor digital de muñeca (Fig. 1C).

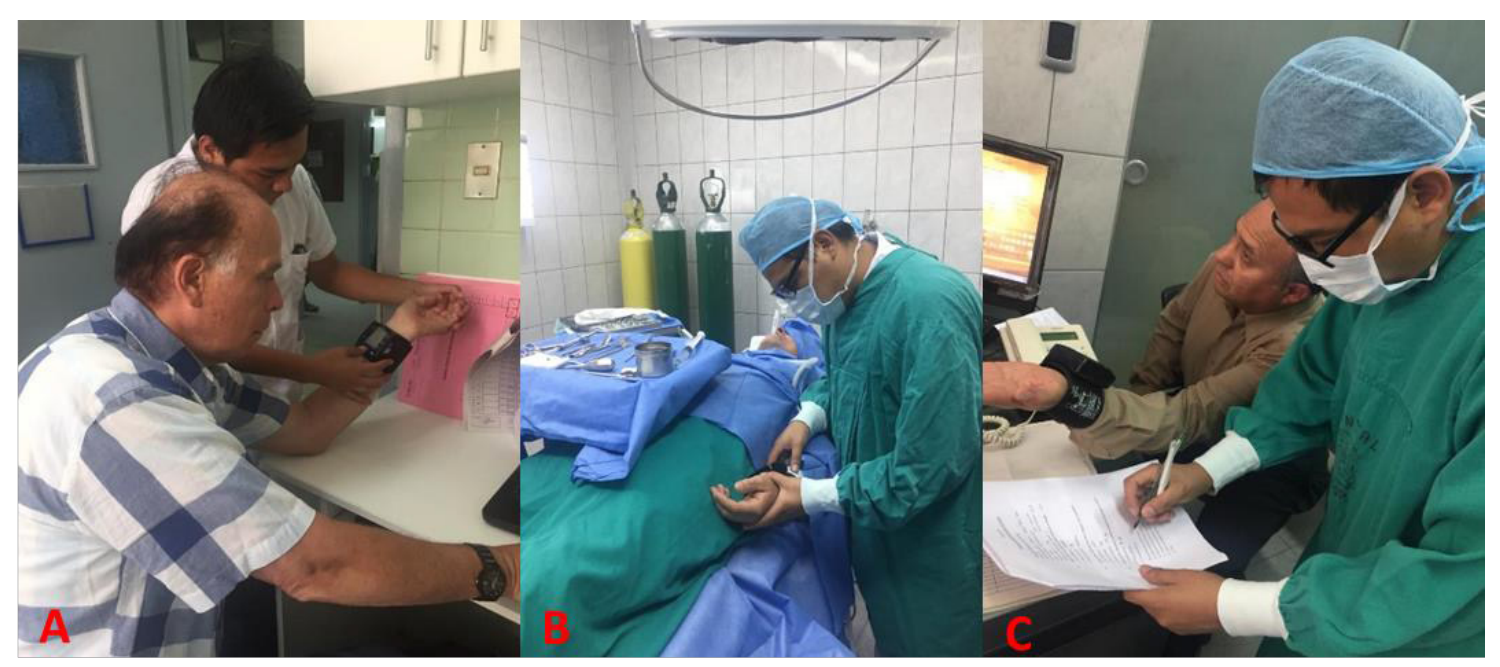

Fig. 1. A. Registro de parámetros antes de la cirugía (preoperatorio). B. Registro cinco minutos luego de iniciado la anestesia local (intraoperatorio). C. Registro luego de cinco minutos de finalizada la cirugía (posoperatorio) 
La comparación entre los datos numéricos y el grupo según edad, sexo y tipo de técnica quirúrgica (colocación de implantes solo y colocación del implante más regeneración ósea guiada) se realizó a través de la t de Student para grupos independientes. La comparación según los tiempos de evaluación se realizó a través de la prueba ANOVA para medidas relacionadas y un análisis post hoc de Bonferroni. Ambas pruebas se realizaron luego de demostrar los supuestos de distribución normal y homogeneidad de medias. Para la refutación de la hipótesis nula se tomó un nivel de significancia de 0,05. El estudio no forzó a cooperar a ningún paciente; cada persona tuvo la elección de escoger libremente para cooperar en la investigación por medio de un consentimiento informado. La utilización del instrumento no generó diferencias de ninguna clase (igualdad). La investigación no publicó nombres ni reportes individuales. Las informaciones que se analizaron se mostraron tal cual se encontró sin faltar a la verdad. El estudio fue aprobado por el Comité de Ética de la Facultad de Medicina Tropical de la Universidad Nacional Mayor de San Marcos, así como del Comité de Ética del Centro Médico Naval “Cirujano Mayor Santiago Távara” (Lima. Perú).

\section{Resultados}

Se evaluaron 26 pacientes con promedio de edad 51,69 $\pm 11,06$ de los cuales el 65,4\% fueron del sexo masculino (promedio de edad de $52,65 \pm 11,59)$ y el $34,6 \%$ del sexo femenino (promedio de edad 49,89 $\pm 10,4$ ).

A cada paciente se le colocó un implante dental de los cuales el $92,3 \%$ fueron tardíos, el 7,7\% fueron inmediatos; en el 73,1\% no se realizó un procedimiento adicional y en el $26,9 \%$ se realizaron procedimientos de regeneración ósea guiada. $\mathrm{Al}$ evaluar las variaciones de la temperatura corporal se encontró que durante el preoperatorio

Tabla 1. Variaciones de los parámetros sistémicos según momentos de evaluación

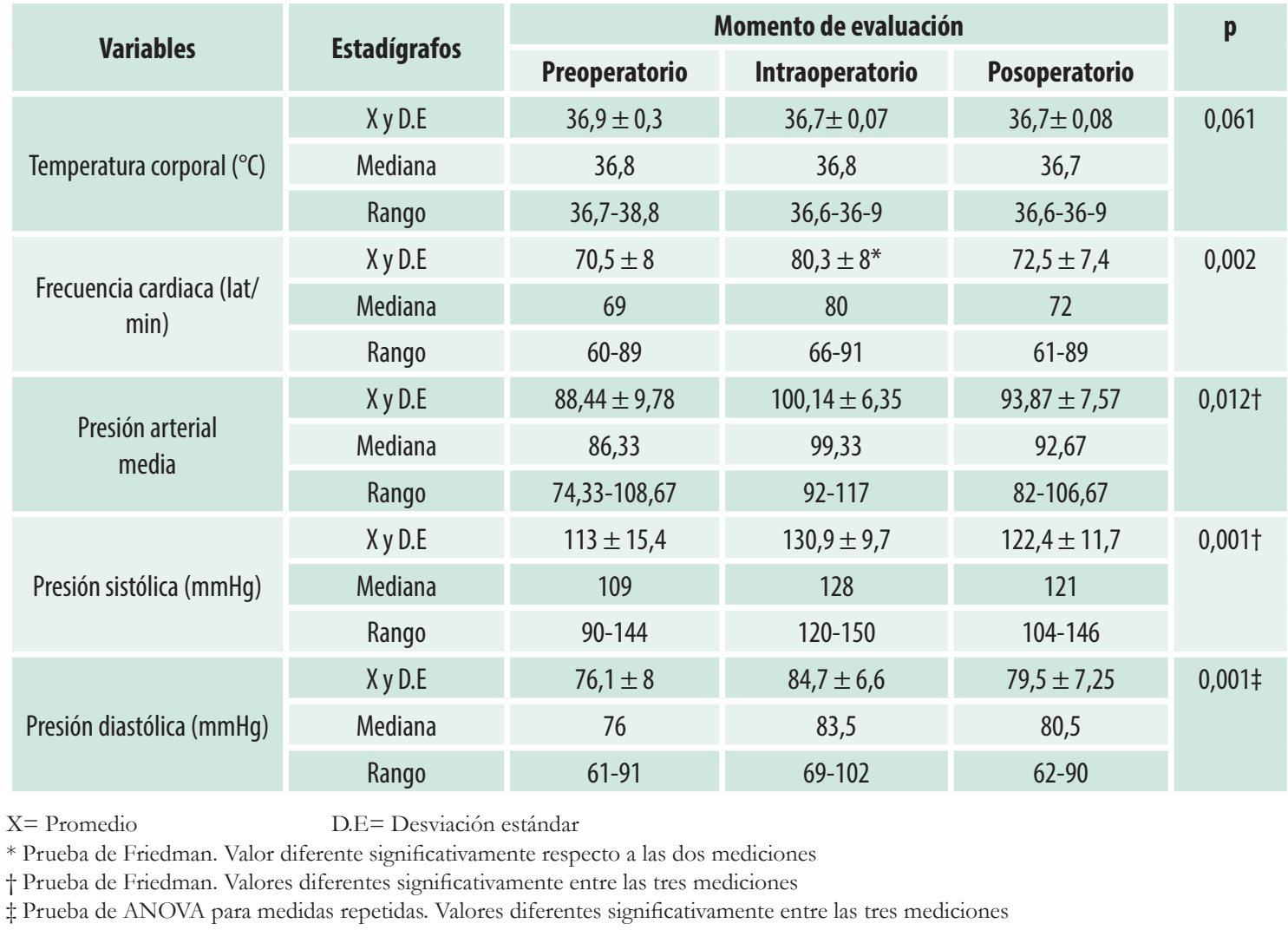


el promedio fue de $36,9^{\circ} \mathrm{C}$ mientras que en el intra y posoperatorio fue similar con un valor de $36,7^{\circ} \mathrm{C}$. La frecuencia cardiaca aumentó durante el intraoperatorio $(80,3$ lat $/ \mathrm{min})$ y se restableció en el posoperatorio $(72,5 \mathrm{lat} / \mathrm{min})$. La presión arterial media en el intraoperatorio en promedio fue $100,14 \mathrm{mmHg}$ mientras que en el posoperatorio fue $93,97 \mathrm{mmHg}$ (Tabla 1 y Fig. 2).

\section{Discusión}

Bajas o altas temperaturas corporales pueden alterar las tasas metabólicas, función de los órganos y causar agravios en los tejidos ${ }^{(7)}$. Los cambios hemodinámicos como frecuencia cardiaca son uno de los parámetros a considerar en la exploración clínica de un paciente, la aparición de una taquicardia o una bradicardia extrema podría dar lugar a consecuencias fatales ${ }^{(8)}$ y un aumento o disminución de la presión arterial está sujeto a factores, conformando en su conjunto un considerable riesgo para la integridad de la persona sino hubiera una idónea atención en la práctica quirúrgica ${ }^{(9)}$.

En el presente trabajo se valoró cómo se modifican estos parámetros en intervenciones quirúrgicas implantológicas, otros estudios evaluaron la presión parcial de oxígeno $\left(\mathrm{SpO}_{2}\right)^{(8,10,11)}$, así como el grado de ansiedad ${ }^{(12)}$ y el carácter del paciente ${ }^{(13)}$. El procedimiento odontológico en el que se evaluó la temperatura, presión arterial y frecuencia cardiaca fue la colocación de implantes al igual que otras investigaciones ${ }^{(6,12-}$ ${ }^{14)}$, otros autores lo hicieron durante exodoncias $(1,9,10,11,15,16)$ en endodoncias ${ }^{(17)}$, y tratamientos odontológicos de rutina (alisado radicular, profilaxis, operatoria e impresiones) ${ }^{(18)}$.

Al estudiar los cambios de la presión arterial media, el presente estudio encontró que se evidenció un incremento significativo de la presión media en los sujetos desde el momento intraoperatorio y si bien disminuyó en el posoperatorio no fue igual al estado basal. Al respecto, Carrasco y cols ${ }^{(6)}$ también hallaron un alza significativa de la presión arterial media

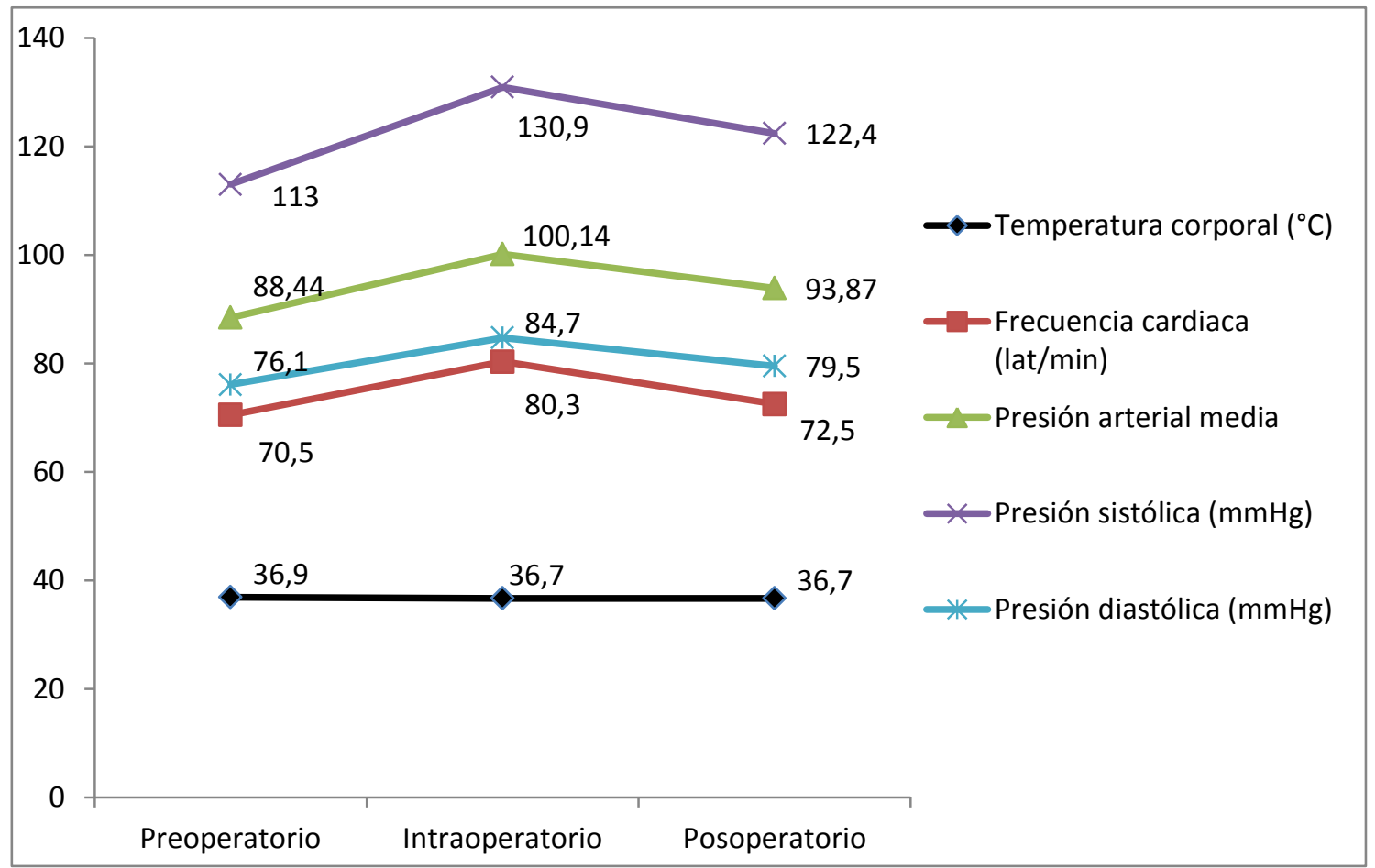

Fig. 2. Variaciones de los promedios según momentos de evaluación 
durante la intervención quirúrgica de implantes; esto se puede deber al estrés quirúrgico que puede aumentar los valores de catecolaminas circulantes y producir un aumento de la presión arterial media ${ }^{(4)}$; sin embargo Masahiro y cols ${ }^{(13)}$ encontraron una correlación del neuroticismo y el aumento de la presión arterial media en personas que recibieron implantes, por su parte Weber y cols ${ }^{(19)}$ informaron que una persona con un alto grado de neuroticismo tiende a seleccionar las situaciones que podrían conducir a la adversidad y la angustia y modificar sus parámetros sistémicos; asimismo Richmond y cols ${ }^{(20)}$ encontraron un incremento de la presión arterial media en personas sujetas a tratamientos dentales de rutina sin anestesia local. Esto puede ser debido que, al realizar tratamientos sin anestésico, exista mayor posibilidad de sentir molestia o dolor. Podemos resaltar que estas variaciones pueden haber aumentado con la edad, esto debido a que a medida que aumenta la edad disminuye la elasticidad de las paredes de las arterias. En personas mayores de 40 años se presenta con mayor frecuencia este incremento ${ }^{(18)}$. Nakamura y cols ${ }^{(21)}$ mencionan que la administración de anestesia local activa el flujo simpático adrenal por lo que podría influir en la elevación de la presión arterial.

Al estudiar los cambios de la presión arterial sistólica y diastólica, el presente trabajo encontró que estos aumentan en el momento intraoperatorio (5 min después de aplicarse la anestesia) $y$ tienen un leve descenso en el posoperatorio, siendo significativamente diferente en el intraoperatorio. Al respecto Nagao y cols ${ }^{(14)}$ encontraron que la presión arterial sistólica aumentó significativamente y la presión arterial diastólica no cambio significativamente en comparación con el control preoperatorio durante toda la cirugía de implantes dentales, Tiwari y cols (10) encontraron un incremento de la presión arterial sistólica durante la administración del anestésico, y un cambio mínimo en la presión arterial diastólica durante una exodoncia dental; Nuñez y cols ${ }^{(15)}$ encontraron que la varia- ción de la presión arterial con más frecuencia fue a los 5 minutos de haber aplicado el anestésico en exodoncias.

En la frecuencia cardiaca, se encontró que existió un aumento significativo del pulso en los pacientes sólo durante el momento intraoperatorio y regresó a su estado basal después de realizada la cirugía, al respecto Tiwari y cols ${ }^{(10)}$ y Matsumura y cols ${ }^{(22)}$ encontraron que hubo un aumento de la frecuencia cardiaca durante la administración de anestesia local en las exodoncias, Arias y cols ${ }^{(8)}$ encontraron que la frecuencia del pulso aumentó de manera significativa después del anestésico y permaneció estable durante el levantamiento del colgajo, volvió a bajar durante la osteotomía, conservándose durante la sutura y el alta en las exodoncias, Nagao y cols ${ }^{(14)}$ encontraron que aumentó justo después de la anestesia local, y bajó en el transcurso de la cirugía de implantes dentales.

Fuentes y cols ${ }^{(5)}$ refieren que las catecolaminas producidas en situaciones de esteres quirúrgico aumentan la presión arterial y la frecuencia cardiaca, sin embargo Vintanel y cols ${ }^{(11)}$ indican que el grado de estrés es una variable a considerar en el inicio de las variaciones sistémicas (frecuencia cardiaca y presión arterial diastólica), Nagao y cols ${ }^{(14)}$ acotan que el aumento de la edad, el tiempo de cirugía y la cantidad de implantes colocados se asocian con mayores variaciones de parámetros sistémicos (frecuencia cardiaca, presión arterial sistólica y diastólica).

Respecto a la temperatura, se encontró que existió una disminución significativa de la temperatura en los pacientes desde el momento intraoperatorio y siguió constante después de realizada la cirugía, aunque clínicamente no fue significante. Al respecto se indica una disminución marcada de la temperatura cuando se utiliza anestesia general y regional, mas no así cuando se utiliza anestesia local ${ }^{(23)}$, sin embargo, Kimberger y Quast ${ }^{(7)}$ indican que la persona suele percibir enfriamiento accidental cuando se inicia el procedimiento quirúrgico. Factores externos como usar pocas prendas 
de vestir o bajas temperaturas en el ambiente inducen la involuntaria la disminución de calor. En el presente trabajo, si bien es cierto que el aire acondicionado suministra entornos agradables y comodidad para el cirujano, resulta un factor externo en su uso durante todos los tratamientos de implantes dentales. El aire acondicionado debe presentar las cualidades técnicas adecuadas ya que hoy en día muchas intervenciones quirúrgicas que se efectúan en los hospitales precisan de un ambiente aséptico. El presente trabajo tuvo como limitante que no pudo evaluarse la presión parcial de oxígeno $\left(\mathrm{PpO}_{2}\right)$ debido a que no se contó con los materiales necesarios para la medición. De esta forma recomendamos considerar esta variable en los diferentes procesos de la etapa quirúrgica puesto que es importante ya que refleja la saturación de la hemoglobina y por tanto el aporte de oxígeno a los tejidos. Solo se valoraron pacientes sanos y que hayan planificado la colocación máxima de dos implantes; se requiere estudios que evalúen pacientes con enfermedades sistémicas (siempre que se respeten las consideraciones bioéticas o la opinión de expertos) y que requieran procedimientos más complejos de colocación de implantes dentales. Cabe indicar que la variable "dolor" es una factor también a considerar en futuros estudios pues podría afectar los valores de presión arterial. Es recomendable analizar el grado de ansiedad que presentan los pacientes para lo cual recomendamos el uso de escalas o test psicométricos antes de colocar un implante dental. Añadimos que para la el tamaño muestra se utilizó como base los principales antecedentes; sin embargo, esta cantidad fue pequeña y no es aplicable la generalización de datos, en ese sentido recomendamos un mayor tamaño de muestra para futuros estudios.

\section{Conclusión}

Existe una disminución no significativa de la temperatura desde el momento intraoperatorio y se vuelve constante hasta finalizar la colocación del implante dental; la frecuencia cardiaca aumenta desde el momento intraoperatorio y si bien disminuye en el posoperatorio no es igual a su estado basal; similar condición cuando se evalúa la presión arterial media en pacientes sanos sometidos a la colocación de uno o dos implantes dentales.

\section{Contribución de autoría}

1. Concepción y diseño del estudio

2. Adquisición de datos

3. Análisis de datos

4. Discusión de los resultados

5. Redacción del manuscrito

6. Aprobación de la versión final del manuscrito

AQA ha contribuido en 1, 3, 5 y 6 . LRO ha contribuido en 2, 4 y 6.

YCR ha contribuido en 2, 4, 5 y 6 .

SGP ha contribuido en 2, 4, 5 y 6.

\section{Referencias bibliográficas}

1. Pando P, Expósito F. Exodoncia dental y riesgo cardiovascular. Variabilidad de las constantes hemodinámicas durante la exodoncia dental. Cient Dent. 2008; 5(3): 175-81.

2. Huang JI, Yu HC, Chang YC. Occurrence of trigeminocardiac reflex during dental implant surgery: An observational prospective study. J Formos Med Assoc. 2017; 116(10): 742-747.

3. Schimmel M, Srinivasan M, McKenna G, Müller $\mathrm{F}$. Effect of advanced age and/or systemic medical conditions on dental implant survival: A systematic review and meta-analysis. Clin Oral Implants Res. 2018; 29(Suppl 16): 311-330.

4. Nogareda C. Fisiología del estrés. [consultado el 15 de abril del 2019]. [Internet]. Available from: http: //www.insht.es/InshtWeb/Contenidos/Documentacion/FichasTecnicas/NTP/ Ficheros/301a400/ntp_355.pdf

5. Nobre Mde A, Maló P, Gonçalves Y, Sabas A, Salvado F. Outcome of dental implants in diabetic patients with and without cardiovascular disease: A 5-year post-loading 
retrospective study. Eur J Oral Implantol. 2016; 9(1): 87-95.

6. Carrasco R, Cordero E, Goldberg I, Martinez B, Pacheco C. Evaluación de la presión arterial media durante la cirugía de implante dental. Rev Esp Cir Oral Maxilofac. 2014; 36(3): 95-8. DOI: http: //dx.doi.org/10.1016/j. maxilo.2013.02.002.

7. Kimberger O, Quast S. La importancia de la temperatura corporal central. 1 ra ed. Alemania. Dräger Medical GmbH; 2016. 9-60 p.

8. Arias B, Romero M, Hita C, Bravo M, Vallecillo M. Seguimiento de la frecuencia cardiaca y la presión parcial de oxígeno durante la cirugía bucal. Av Odontoestomatol. 2003; 19(2): 1-6.

9. Castillo C, Manotas A, Vargas D. Variabilidad de la presión arterial pre y post-quirúrgica en pacientes sometidos a cirugía oral que asistieron a la clínica odontológica de la Universidad del Magdalena en los meses de abril y mayo periodo 2008. Duazary. 2011; 8(1): 1-10.

10. Tiwari V, Sridhar M, Teja P, Rama K, Tiwari $\mathrm{H}$. Evaluation of systolic and diastolic blood pressure, pulse rate and spo 2 levels pre and post dental extraction under local anesthesia. J Oral Med. 2018; 4(2): 74-8.

11. Vintanel M. Influencia del anestésico, ansiedad e hipertensión en los cambios hemodinámicos de pacientes sometidos a extracción dental. [tesis de licenciatura]. [Madrid]: Facultad de Odontología, Universidad Complutense de Madrid; 2015. 57p.

12. Schwartz D, Bar Y, Ilana E. Efecto del estrés en el procesamiento de la información en el contexto de la cirugía de implante dental. Clin Oral Implants Res. 2006; 1: 1-8.

13. Masahiro W, Syunta M, Tomoaki M, Tohru S, Kazunori I, Yoshinobu M. Un estudio prospectivo de la relación entre el carácter del paciente y la presión arterial en la cirugía de implantes dentales. Rev Int Implantol. 2016; 2(21): $1-5$.

14. Nagao H, Munakata M, Tachikawa N, Shiota M. [Clinical study of risk management for dental implant treatment--changes of blood pressure and pulse rate during implant surgery under local anesthesia].[Article in Japanese] Kakubyo Gakkai Zasshi. 2002; 69(1): 27-33.

15. Núñez M, Tore A, Mico G, Pratt G, Pratt G, Keim M. Variabilidad de la presión arterial en pacientes normotensos sometidos a cirugía bucal ambulatoria. Rev Fac Cienc Salud UDES. 2015; 2(2): 125-32.

16. Gungormus M, Buyukkurt M. La evaluación de los cambios en la presión arterial y la frecuencia del pulso de los pacientes hipertensos durante la extracción dental. Acta Med Austriaca. 2003; 30(5): 127-9.

17. Macías A, Oliver P, Luna L, Guerrero A, Cornejo P. Efecto del tratamiento endodóntico en los valores de la presión arterial en pacientes hipertensos. Rev ADM. 2013; 70(1): 30-4.

18. Aranzazu M, Delgado J, Pieschacón G. Variaciones de riesgo en valores de tensión arterial en pacientes hipertensos durante procedimientos odontológicos. Rev la Univ Ind Santander. 2014; 46(2): 1-10.

19. Weber M, Schiffrin E, White W, Mann S, Lindholm L, Kenerson J, y cols. Clinical practice guidelines for the management of hypertension in the community. J Clin Hypertens. 2014; 16(1): 14-26. doi: 10.1097/ HJH.0000000000000065.

20. Richmond A, Gortzack D, Johannes O, Abraham I. Respuesta de la presión arterial al tratamiento dental de restauración de rutina con y sin anestesia local: registro continuo no invasivo de la presión arterial con un manómetro para dedos. Cir Oral. 1992; 73(6): 677-81.

21. Nakamura Y, Matsumura K, Miura K, Kurokawa H, Abe I, Takata Y. Cardiovascular and sympathetic responses to dental surgery with local anesthesia. Hypertens Res. 2001; 24(3): 209-14.

22. Matsumura K, Miura K, Takata Y, Kurokawa $\mathrm{H}$, Kajiyama M, Abe I, y cols. Changes in Blood Pressure and Heart Rate Variability During Dental Surgery. Am J Hypertens. 1998; 1: 1376-80.

23. Uriostegui S, Nava L, Mendoza E. Alteraciones de la temperatura y su tratamiento en el perioperatorio. Rev Mex Anestesiol. 2017; 40(1): 29-37.

\section{Yuri Castro Rodríguez: yuricastro_16@hotmail.com}

